\title{
Interconnection Mechanism of Ni-Reinforcement Particle Filled Solderable Polymer Composites with Low-Melting-Point Alloy Filler
}

\author{
Hee Jun Youn ${ }^{1}$, Jeong Il Lee ${ }^{1}$, Jong-Min Kim ${ }^{1, *}$ and Byung-Seung Yim²,* \\ ${ }^{1}$ School of Mechanical Engineering, Chung-Ang University, Seoul 156-756, Korea \\ ${ }^{2}$ School of Mechanical System Engineering, Kangwon National University, Gangwon-do 25913, Korea
}

A novel bonding material system and its interconnection mechanism using a Ni-reinforcement particle filled solderable polymer composites (SPCs) with a low-melting-point alloy (LMPA) filler was proposed to enhance the mechanical properties of the SPC joints. To confirm the feasibility of the proposed interconnection mechanism of Ni-reinforcement particle filled SPC, two types of wetting test (e.g., flat and line pattern wetting test) were conducted. The Ni-reinforcement particle filled SPC exhibited better wettability compared with those of SPC without $\mathrm{Ni}$ particle. In the microstructure inspection, ternary $\mathrm{Cu}-\mathrm{Ni}-\mathrm{Sn}$ intermetallic compound (IMC) was formed at surface of the Ni particles within the LMPA region and bonding interface between $\mathrm{Cu}$ metallization and LMPA. Additionally, the flow, coalescence and selective wetting behaviors of molten LMPA filler was not hindered by incorporated Ni particles. [doi:10.2320/matertrans.MT-M2020265]

(Received August 18, 2020; Accepted September 28, 2020; Published October 30, 2020)

Keywords: electrically conductive adhesive (ECA), low-melting-point alloy, polymeric composites, reinforcement particle, selective wetting

\section{Introduction}

Solderable polymer composites (SPCs) mainly consisted of a polymer composite with reduction capability and a fusible filler have been introduced to overcome the limitations of conventional non-fusible filler-filled electrically conductive adhesives (ECAs). This bonding material system combines the merits of solder materials (i.e., metallurgical interconnection) and conventional non-fusible filler-filled ECAs (i.e., low processing temperatures and simplification of the whole processes). In our previous works, we proved that SPCs can achieve higher electrical and mechanical bonding properties and thermomechanical reliability than conventional ECAs via the metallurgical conduction path formation by the molten fillers. ${ }^{1-3)}$ In the SPC system, the $\mathrm{Sn} / 58 \mathrm{Bi}$ eutectic solder powder with a melting temperature $\left(T_{m}\right)$ of $412 \mathrm{~K}$ has normally been used as a low-melting-point alloy (LMPA) fillers to achieve excellent metallurgical conduction paths through the proper flow, coalescence and wetting behaviors of the molten fillers before the polymer curing. Nevertheless, SPCs with $\mathrm{Sn} / 58 \mathrm{Bi}$ filler have several critical issues, including poor ductility of joint due to the embrittling nature of $\mathrm{Bi}^{4)}$ and decreased reliability due to $\mathrm{Bi}$ segregation or coarsening and excessive growth of IMC layer at the bonding interface. ${ }^{5)}$

To improve the bonding properties of $\mathrm{Sn} / 58 \mathrm{Bi}$ eutectic solders, micro-/nanometer sized metallic reinforcement particles (e.g., $\left.\mathrm{Ag},{ }^{5)} \mathrm{Cu},{ }^{6}\right) \mathrm{In}^{7)} \mathrm{Ni}^{8)}$ etc.) have been incorporated into the solder matrix and formed composite solder. However, the research about metallic reinforcement particles incorporated to SPC system have not been reported.

In this paper, we propose a novel bonding material system and its interconnection mechanism using a reinforcement particle filled SPC with an LMPA filler to enhance the mechanical properties of the SPC joints. As shown in Fig. 1, a metallurgical conduction path is mainly established by the flow-coalescence-wetting behaviors of molten LMPA fillers,

*Corresponding authors, E-mail: 0326kjm@cau.ac.kr, bsyim@kangwon. ac. $\mathrm{kr}$ (a)

(b)

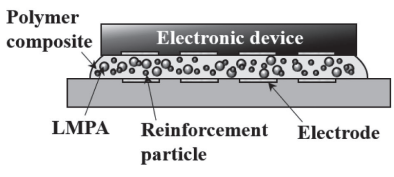

(c)

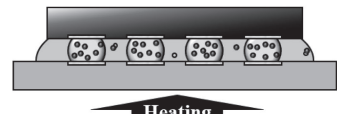

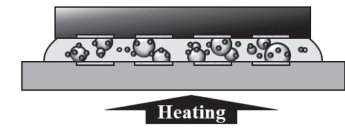

(d)

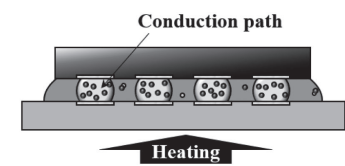

Fig. 1 Schematic of conduction path formation mechanism of the Nireinforcement particle filled SPCs with LMPA filler. (a) Initial condition, (b) flow-coalescence-wetting behaviors of molten LMPA filler, (c) conduction path formation and (d) completion of interconnection process by the polymer curing.

and metallic reinforcement particles are incorporated into conduction path during the reflow process. As the reinforcement particles incorporated into the conduction path formed by LMPA, the bonding properties (e.g., wettability, strength, ductility, fracture toughness, creep resistance, thermomechanical reliability, etc.) of the SPC joints can be enhanced because of a suppression of grain boundary sliding by the dispersion strengthening effect, change in the intermetallic compound formation and uniform stress redistribution. ${ }^{9,10)}$ Among the metallic particle, $\mathrm{Ni}$ was chosen as the reinforcement particle in this study because it has proper wettability with $\mathrm{Sn}$ and it easily forms intermetallics with Sn. ${ }^{10)}$

To investigate the feasibility of the proposed interconnection mechanism using Ni-reinforcement particle filled SPC, two kinds of wetting test (e.g., flat pattern and line pattern wetting test) were conducted using the formulated SPCs.

\section{Experimental}

As can be seen in Table 1, the Ni-reinforcement particle filled SPCs mainly composed of a polymer composite, LMPA 
Table 1 Components of Ni-reinforcement particle filled SPC formulations.

\begin{tabular}{|c|c|c|}
\hline & omponents & Detail \\
\hline \multirow{4}{*}{$\begin{array}{c}\text { Polymer } \\
\text { Composite }\end{array}$} & Base Resin & DGEBA \\
\hline & Curing Agent & DDM \\
\hline & Catalyst & $\mathrm{BF}_{3} \mathrm{MEA}$ \\
\hline & Reductant & Carboxylic Acid \\
\hline \multirow{2}{*}{ Filler } & LMPA & $\mathrm{Sn}-58 \mathrm{Bi}(\varphi: 45 \mu \mathrm{m})$ \\
\hline & Reinforcement Particle & $\mathrm{Ni}(\varphi: 10 \mu \mathrm{m})$ \\
\hline
\end{tabular}

solder fillers and Ni particles. For the polymer composite formulation, DGEBA (diglycidyl ether of bisphenol A; Kukdo Chemical) was used as the base resin, and DDM (curing agent: 4,4'-diaminodiphenylmethane; TCI Korea), $\mathrm{BF}_{3} \mathrm{MEA}$ (catalyst: boron trifluoro-mono-ethylamine; Wako Pure Chemical) and carboxylic acid (reductant; Aldrich Chemical) were used as the minor organic additives. A Sn/ 58Bi eutectic solder powder (Heraeus) with a particle diameter of $45 \mu \mathrm{m}\left(T_{m}=412 \mathrm{~K}\right)$ and Ni particle (Probes) with a diameter of $10 \mu \mathrm{m}$ were used as the LMPA filler and reinforcement particle, respectively. After the polymer matrix formulation was completed, LMPA fillers and Ni particles were mechanically blended to ensure uniform distribution.

In this study, to investigate the feasibility of the proposed interconnection mechanism using Ni-reinforcement particle filled SPC, two types of wetting test were performed. To measure the Ni particle effect on the wettability of LMPA for the metallization, a flat pattern wetting test for a flat-type Cu pattern $(16.0 \mathrm{~mm} \times 16.0 \mathrm{~mm} \times 0.018 \mathrm{~mm}$ in size $)$-formed test coupon was conducted. For the flat pattern wetting test, SPC with a filler volume fraction of $50 \%$ (mixing ratio between LMPA and $\mathrm{Ni}$ particle (vol\%): 95:5) was formulated. The formulated SPC was mounted onto the cleaned flat-type $\mathrm{Cu}$ pattern, and then reflowed according to the temperature profile; the test vehicle was heated from room temperature to $433 \mathrm{~K}$ at a ramped heating rate of $38 \mathrm{~K} / \mathrm{min}$ to melt the LMPA fillers, and then the temperature was maintained at $433 \mathrm{~K}$ for $3 \mathrm{~min}$ to cure the polymer composite. After the flat pattern wetting test was completed, the wetting angle of the LMPA for the metallization was measured using an optical microscope (VHX-1000; Keyence) and compared to those of SPC without $\mathrm{Ni}$ particle. Additionally, microstructure of wetted LMPA was inspected using a field-emission scanning electron microscope (FESEM; Sigma; Carl Zeiss Co. Ltd.), and their chemical composition was identified using an energy-dispersive spectrometer (NORAN System 7; Thermo Scientific Co.).

During the interconnection process using Ni-reinforcement particle filled SPCs, to ensure the ideal conduction path formation by LMPAs and effective reinforcing effect by $\mathrm{Ni}$ particle, LMPA fillers and Ni particles within the SPC should be sufficiently concentrated onto the metallization region by the flow-coalescence-wetting behaviors of the molten filler. To investigate the selective concentration behavior of LMPA fillers and $\mathrm{Ni}$ particles for metallization region, a line pattern wetting test for a line-type $\mathrm{Cu}$ pattern $(5.0 \mathrm{~mm} \times 0.1 \mathrm{~mm} \times$ $0.018 \mathrm{~mm}$ in size, $300 \mu \mathrm{m}$ in pattern pitch)-formed test coupon was conducted. For the line pattern wetting test, formulated SPC filled with a filler volume fraction of $30 \%$
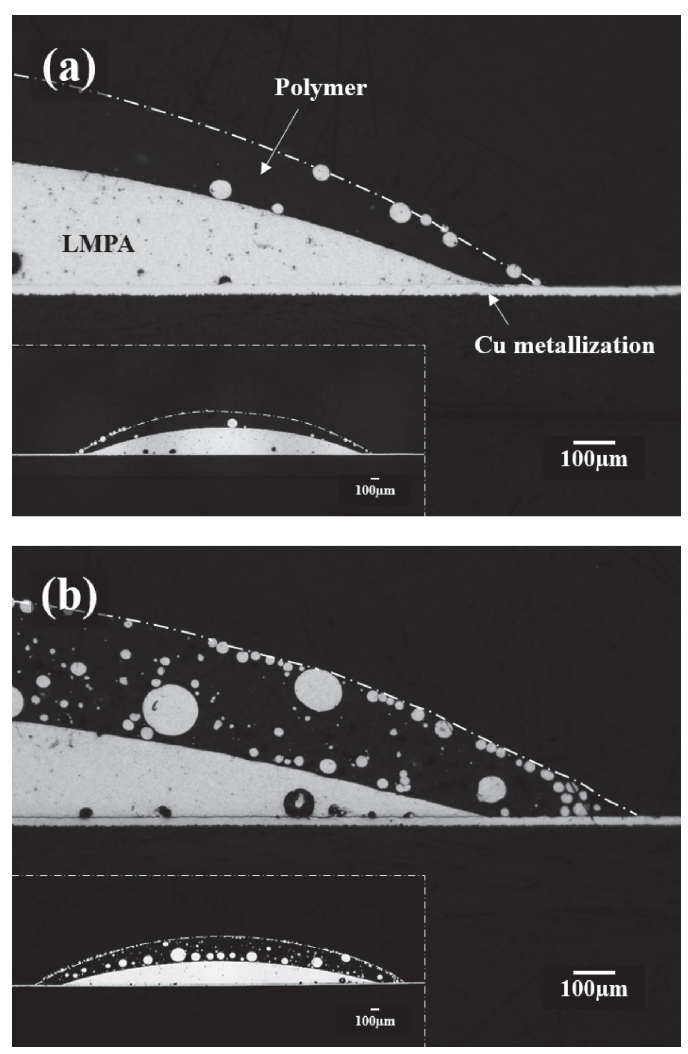

Fig. 2 Wetting morphology of the LMPA filler for the $\mathrm{Cu}$ metallization. (a) SPC without Ni particles and (b) with Ni particles.

(mixing ratio between LMPA and Ni particle (vol\%): 95:5) was uniformly deposited on entire testing area (including $\mathrm{Cu}$ pattern and PCB regions) of the cleaned test coupon. Then, transparent glass board was placed over the deposited SPC layer to investigate the flow-coalescence-wetting behavior of the filler during reflow process; the standoff height between the test coupon and glass board was maintained by the copper wires with a diameter of $100 \mu \mathrm{m}$. The prepared test coupon was then reflowed.

\section{Results and Discussion}

Figure 2 shows the wetting morphology of LMPA filler on the flat-type $\mathrm{Cu}$ pattern for the SPC with and without $\mathrm{Ni}$ particles. The wetting regions have two definite regions; one is a wetted LMPA region by the coalescence and wetting behaviors of molten LMPA fillers, and the other is a cured polymer region wrapped around the wetted LMPA. As shown in the results, the wettability of molten LMPA was improved by adding the Ni particle for the SPC; i.e., the LMPA within the Ni-reinforcement particles filled SPC more widely spread than LMPA within the SPC without Ni particles, and the wetting angle of Ni-reinforcement particles filled SPC (approximately $15.1 \mathrm{deg}$ ) was also lower than that of SPC without $\mathrm{Ni}$ particles (approximately $20.0 \mathrm{deg}$ ). Such an improved wettability of LMPA for $\mathrm{Cu}$ pattern may be attributed to decreased surface tension and increased fluidity of molten LMPA solder material by adding Ni particles. ${ }^{11,12)}$

The microstructure inspection results of wetted LMPA filler for the $\mathrm{Cu}$ metallization are shown in Fig. 3. Figure 3(a) shows the morphology of Ni particle within the LMPA. As 

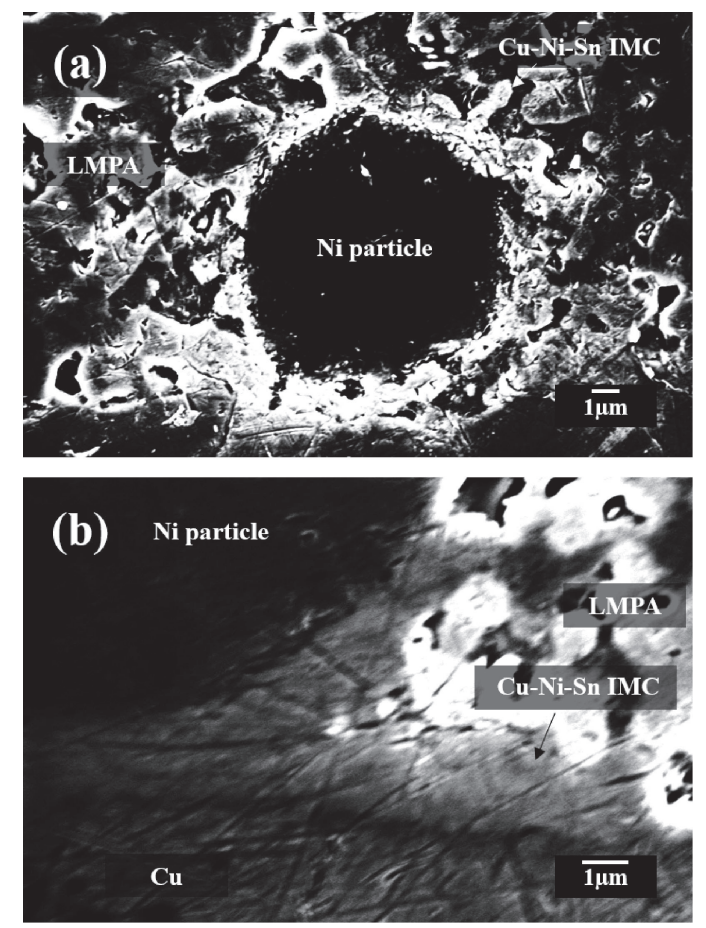

Fig. 3 Microstructure of the Ni-reinforcement particle filled SPC joint. (a) Ni-reinforcement particle and the IMC layer formed around Ni particle and (b) interfacial microstructure between $\mathrm{Cu}$ metallization and LMPA.

can be seen in the figure, the sunflower-type ternary $\mathrm{Cu}-\mathrm{Ni}-$ $\mathrm{Sn}$ intermetallic compound layer (IMC) with a thickness of $1 \sim 3 \mu \mathrm{m}$ was formed around the Ni particle. When the cracks propagate within the solder joint, the cracks are not propagated through the reinforcement particles and flow around the particles. Such a propagated crack around the particle is disturbed by the sunflower-type IMC and thus crack propagation is retarded by these obstacles. ${ }^{13)}$ Therefore, these sunflower-type IMC around the $\mathrm{Ni}$ particles can improve the mechanical properties of solder joint because of the strengthening effect from their IMC morphology.

Figure 3(b) shows the interfacial microstructure between the $\mathrm{Cu}$ metallization and LMPA solder material. As can be observed in the figure, a metallurgically interconnected scallop-shaped ternary $\mathrm{Cu}-\mathrm{Ni}-\mathrm{Sn}$ IMC with a thickness of $2 \sim 5 \mu \mathrm{m}$ was formed between the $\mathrm{Cu}$ metallization and LMPA. During reflow process, $\mathrm{Ni}$ atoms can alter the $\mathrm{Cu}-\mathrm{Sn}$ IMC composition in the bonding interface by substituting the $\mathrm{Cu}$ atoms in the IMC lattice and forming a $\mathrm{Cu}-\mathrm{Ni}-\mathrm{Sn}$ IMC. This $\mathrm{Cu}-\mathrm{Ni}-\mathrm{Sn}$ IMC is more thermodynamically stable than the $\mathrm{Cu}-\mathrm{Sn} \mathrm{IMC}$, and thus can improve the long-term reliability of electronic joints due to hinder the excessive brittle IMC layer growth. ${ }^{8,14)}$

Figure 4 shows the in situ selective concentration behavior of LMPA fillers and Ni particles for metallization region by the flow-coalescence-wetting behaviors of the filler within the formulated SPC for the line-type $\mathrm{Cu}$ pattern-formed wetting test coupon. The LMPAs and Ni particles were uniformly distributed within the SPC at the initial conditions (Fig. 4(a)). As can be seen in Fig. 4(b), after reflow process, the LMPAs were concentrated on the line-type $\mathrm{Cu}$ pattern. During the reflow process, as increasing reflow temperature, the molten LMPA fillers coalesced with nearby filler due to internal flow
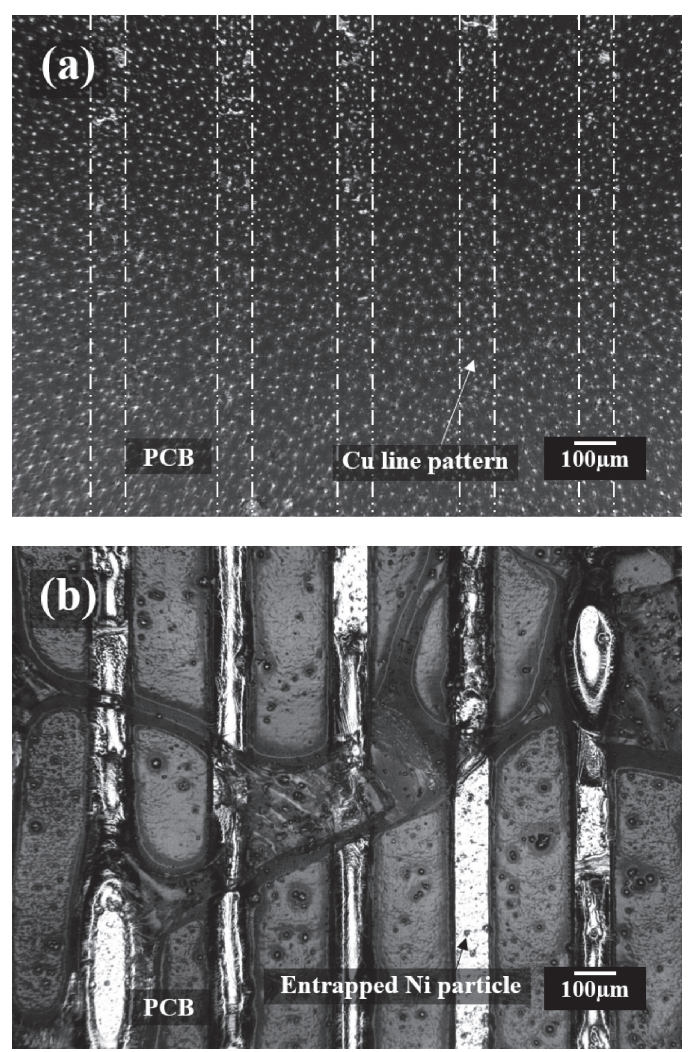

Fig. 4 Wetting morphology of molten fillers within Ni-reinforcement particle filled SPCs for the line-type $\mathrm{Cu}$ pattern. (a) Initial condition and (b) after reflow process.

and contact within the polymer composite, and Ni reinforcement particles were also entrapped into the largely grown LMPA filler by contact and integration. The coalesced molten LMPA fillers selectively wetted on the $\mathrm{Cu}$ line patterns due to the difference in wettability between the substrate and metallization. Through these selective wetting behavior of the molten LMPA fillers, the LMPA and metallic reinforcement particles within the SPC were concentrated onto the $\mathrm{Cu}$ line pattern. Finally, the interconnection process was finished by complete curing of the polymer composite. Although some spherical LMPA fillers and Ni particles remained within the cured polymer region, it did not affect near the $\mathrm{Cu}$ pattern because of the insulation properties of the polymer.

From these results, it can be expected that the Ni particles within the SPC joints can enhance not only the solderability (i.e., enhanced wettability) but also mechanical bonding properties and reliability of SPC joints. Additionally, the flow-coalescence-wetting behaviors of molten LMPA filler is not hindered by additionally incorporated Ni particles, and Ni particles can be supplied to the conduction path region via the selective wetting behavior of molten LMPA filler.

\section{Conclusion}

In this study, novel Ni-reinforcement particle filled SPC was fabricated, and their feasibility was investigated to enhance the bonding properties and reliability of SPC joints. In a flat pattern wetting test, the LMPA using Ni-reinforcement particle filled SPCs showed more improved wettability compared that of SPCs without Ni particles. The sunflower- 
type ternary $\mathrm{Cu}-\mathrm{Ni}-\mathrm{Sn} \mathrm{IMC}$ was formed at surface of the $\mathrm{Ni}$ particles within the LMPA; these sunflower-type IMC around the $\mathrm{Ni}$ particles can improve the mechanical properties of SPC joints because that may act as an obstacle to retard the crack propagation. Additionally, the thermodynamically stable scallop-type ternary $\mathrm{Cu}-\mathrm{Ni}-\mathrm{Sn}$ IMC was formed at the bonding interface between $\mathrm{Cu}$ metallization and LMPA by adding Ni particles.

In a line pattern wetting test, the LMPA and Ni particles within the SPC were concentrated onto the $\mathrm{Cu}$ line pattern and formed a stable metallurgical conduction path through the proper internal flow, coalescence and selective wetting behaviors of the molten filler within the SPCs.

From the results of the present study, we confirmed the feasibility of the newly proposed interconnection mechanism using a Ni-reinforcement particle filled SPC. Base on this interconnection mechanism, we expect that the Ni-reinforcement particle filled SPCs may be able to improve the bonding properties and reliability of SPC joints. A further investigation into the bonding properties and reliability of $\mathrm{Ni}$ reinforcement particle filled SPC joints with LMPA fillers will be performed and reported in the near future.

\section{Acknowledgements}

This work was supported by the National Research Foundation of Korea (NRF) grant funded by the Korea government (MSIT) (No. 2020R1F1A1075165) and 2018 Research Grant from Kangwon National University.

\section{REFERENCES}

1) B.S. Yim, S.H. Oh, J.S. Jeong and J.M. Kim: J. Compos. Mater. 47 (2013) 1141-1152.

2) B.S. Yim, J.M. Kim, S.H. Jeon, S.H. Lee, J. Kim, J.G. Han and M. Cho: Mater. Trans. 50 (2009) 2649-2655.

3) B.S. Yim, J.I. Lee, Y. Heo, J. Kim, S.H. Lee, Y.E. Shin and J.M. Kim: Mater. Trans. 53 (2012) 2104-2110.

4) F. Hua, Z. Mei and J. Glazer: Proc. 48th IEEE Electronic Components and Technology Conference 47, (1998) pp. 277-283.

5) M. McCormack, H.S. Chen, G.W. Kammlott and S. Jin: J. Electron. Mater. 26 (1997) 954-958.

6) H. Takao, A. Yamada and H. Hasegawa: R\&D Rev. Toyota CRDL 39 (2004) 49-56.

7) X. Chen, F. Xue, J. Zhou and Y. Yao: J. Alloy. Compd. 633 (2015) 377-383.

8) A.K. Gain and L. Zhang: J. Mater. Sci. Mater. Electron. 27 (2016) 781794.

9) H.T. Lee and Y.H. Lee: Sci. Technol. Weld. Joining 10 (2005) 353-360

10) P. Yao, P. Liu and J. Liu: J. Alloy. Compd. 462 (2008) 73-79.

11) Y. Wang, Z. Fang, N. Ma and K. Zhang: J. Mater. Sci. Mater. Electron. 28 (2017) 94-99.

12) T. Ventura, C.M. Gourlay, K. Nogita, T. Nishimura, M. Rappaz and A.K. Dahle: J. Electron. Mater. 37 (2008) 32-39.

13) H. Rhee, F. Guo, J.G. Lee, K.C. Chen and K.N. Subramanian: J. Electron. Mater. 32 (2003) 1257-1264.

14) A.T. Tan, A.W. Tan and F. Yusof: Sci. Technol. Adv. Mater. 16 (2015) 033505 . 